\title{
Die Mitarbeiter
}

Afflerbach, Holger, Dr., Hist. Seminar II, Universitätsstr. 1, 40225 Düsseldorf (266)

Angelow, Jürgen, Dr., Platz der Einheit 5, 14467 Potsdam $(129,260,272)$

Arenth, Joachim, Dr., Ortlerstr. 1, 81373 München (97)

Bischof, Günter, Dr., Eisenhower Center, University of New Orleans, New Orleans, LA 70148, USA $(237,309)$

Boog, Horst, Dr., Ringstr. 16, 79252 Stegen $(217,286,287)$

Brüning, Rainer, Dr., Seestraße 61, Haus 4, 71638 Ludwigsburg (257)

Bungert, Heike, M.A., Anglo-Amerikanische Abt., Universität zu Köln, Albertus-MagnusPlatz, 50923 Köln (228)

Burchardt, Lothar, Prof. Dr., Universität Konstanz, Postfach 5560, 78464 Konstanz (249)

Deist, Wilhelm, Prof. Dr., Siegelsbachstr. 3, 79117 Freiburg (194, 263, 268)

Dirks, Uwe, Kapitän z.S., Haager Weg 130, 53127 Bonn (196)

Fesser, Gerd, Dr., Ebertstraße 5, 07743 Jena (273)

Fischer, Alexander, Prof. Dr. ( $\dagger)(288,308)$

Förster, Jürgen, Dr., Wiss. Direktor, MGFA (289)

Förster, Stig, Prof. Dr., Universität Bern, Historisches Institut, Unitobler, Länggassstr. 49, CH-3000 Bern 9 (61, 192)

Franke, Peter, Ministerialrat, Ministerium für Wirtschaft, Mittelstand und Technologie des Landes Nordrhein-Westfalen, Haroldstr. 4, 40213 Düsseldorf (269)

Fröhlich, Michael, Dr., Postfach 150 150, 53040 Bonn $(190,268)$

Fuchs, Konrad, Prof. Dr., Ebersheimer Weg 38a, 55131 Mainz (281)

Gotthard, Axel, Dr., Alexander-Universität Erlangen-Nürnberg, Institut für Geschichte, Kochstr. 4, 91054 Erlangen (251)

Greiner, Christian, Dr., Brandenburger Str. 13, 79211 Denzlingen (256, 311)

Hass, Gerhart, Prof. Dr., Weinbergweg 20, 15834 Rangsdorf $(213,235)$

Heideking, Jürgen, Prof. Dr., Anglo-Amerikanische Abt., Universität zu Köln, AlbertusMagnus-Platz, 50923 Köln $(209,212)$

Heinemann, Winfried, Dr., Major, MGFA (270, 282, 307, 319)

Herwig, H. Holger, Prof., The Univ. of Calgary, 2500 University Drive N.W., Calgary, Alberta, Canada T2N 1N4 (198)

Heyden, Ulrich van der, Dr., Forschungsschwerpunkt Moderner Orient, Prenzlauer Promenade 149-151, 13189 Berlin $(254,262,279)$

Hiller, Günter, Postfach 1525, 61348 Homburg v.d.H. (293)

Kaiser, Stephan, Dr., Wilhelmstr. 1, 56112 Lahnstein (252)

Kehrig, Manfred, Dr., Ltd. Archivdirektor, Oberst a.D. und d.R., Leiter Bundesarchiv-Militärarchiv, Wiesentalstr. 10, 79115 Freiburg (173)

Kletzin, Birgit, Dr., Historisches Institut, Universität Potsdam, Postfach 6015 53, 14415 Potsdam (278)

Krautkrämer, Elmar, Prof. Dr., Neuhäuser Str. 58, 79199 Kirchzarten (296)

Kroener, Bernhard R., Dr., Privatdozent, Wiss. Oberrat, MGFA $(176,181)$

Larres, Klaus, Queen's University of Belfast, Department of Politics, Belfast BT7 1NN, Northern Ireland (301)

Lindner, Rainer, M.A., Universität Tübingen, Institut für Osteuropäische Geschichte und Landeskunde, Wilhelmstraße 36, 72074 Tübingen (186)

Lutz, Karl-Heinz, Dr., Hauptmann, MGFA $(283,295)$

Mauch, Christof, Dr., Angloamerikanische Abteilung, Historisches Seminar, Universität zu Köln, Albertus-Magnus-Platz, 50923 Köln (232)

Mecklenburg, Wolfgang, M.A., Peter-Thumb-Weg 19, 79271 St. Peter/Schwarzwald (290)

Meyer, Georg, Dr., Wiss. Direktor, MGFA (246)

Moll, Martin, Dr., Wilhelm-Kienzl-Gasse 33, A-8010 Graz (275, 319) 
Müller, Winfried, Prof. Dr., Historisches Institut, Universität München, Schellingstr. 10/V, 80799 München $(215,292)$

Neitzel, Sönke, Dr. phil., Historisches Seminar Abt. IV, Johannes-Gutenberg-Universität Mainz, Saarstr. 21, 55099 Mainz (261)

Neumann, Ronald, Dr., Akademie der Wissenschaften und der Literatur, GeschwisterScholl-Str. 2, 55131 Mainz (1)

Nicklas, Thomas, Dr., Friedrich-Alexander-Universität, Institut für Geschichte, Kochstraße 4, 91054 Erlangen (180)

Oppelland, Torsten, Dr., Schrödinger Str. 69, 07745 Jena (264)

Ostermann, Christian F, M.A., 621 Maryland Avenue NE, Washington, D.C. 20002, USA (315)

Otto, Helmut, Prof. Dr., Bleicheroder Str: 15 c, 13187 Berlin (271)

Overesch, Manfred, Prof. Dr., Johanna-Kirchner-Str. 15, 31139 Hildesheim (306)

Overmans, Rüdiger, Dr., Oberstleutnant, MGFA $(241,298,316)$

Pröve, Ralf, Dr., Humboldt-Universität zu Berlin, Lehrstuhl f. Geschichte Preußens, Unter den Linden 6, 10099 Berlin $(178,258)$

Rohkrämer, Thomas, Prof. Dr., University of Auckland, Department of History, Private bag, Auckland, Neuseeland (249)

Rupieper, Hermann-Josef, Prof. Dr., Martin-Luther-Universität, Institut für Geschichte, Postfach 8, 06099 Halle $(274,302)$

Sauer, Thomas, Dr., Universität Tübingen, Seminar f. Zeitgeschichte, Wilhelmstr. 36, 72074 Tübingen (226)

Schneidmüller, Bernd, Prof. Dr., Universität Bamberg, Mittelalterliche Geschichte, 96045 Bamberg (175)

Schönsee, Lutz, Fallersleber-Tor-Wall 3, 38100 Braunschweig (252)

Scholl, Lars U., Dr., Deutsches Schiffahrtsmuseum, Van-Ronzelen-Str., 27568 Bremerhaven (258)

Seier, Hellmut, Prof. Dr., Beethovenstr. 8, 35043 Marburg $(171,184)$

Senner, Martin, Dr., Johannes Gutenberg-Universität Mainz, Fachbereich 16, Geschichtswissenschaft, Historisches Seminar IV, 55099 Mainz (31)

Stegemann, Bernd, Dr., Unterstad 56, 79353 Bahlingen (320)

Steinert, Marlis G., Prof. Dr., 12, av. Adrien Jeandin, CH-1226 Thonex (284)

Steininger, Rolf, Prof. Dr., Institut für Zeitgeschichte, Innrain 52, A-6020 Innsbruck (299, 300)

Steinlechner, Wolfgang, Dr., Leitender Regierungsdirektor, Postfach 9669, 76131 Karlsruhe (238)

Stübig, Heinz, Prof. Dr., Birkenweg 3, 35041 Marburg (303)

Syring, Enrico, Dr., Allendorferstr. 8, 35469 Climbach $(219,221)$

Theiler, Olaf, M.A., Naumburger Str. 42, 12057 Berlin (311)

Thöndl, Michael, Dr., Grenzackerstraße 7-11/19/8, A-1100 Wien (230)

Thoß, Bruno, Dr., Wiss. Oberrat, MGFA $(201,204,318)$

Thun-Hohenstein, Romedio Graf v., Dr., 24256 Salzau (223)

Umbreit, Hans, Dr., Ltd. Wiss. Direktor, MGFA (297)

Vad, Erich, Dr., Reaumurstr. 21, 53125 Bonn (250)

Vogt, Arnold, Prof. Dr., Brandiser Str. 3, 04315 Leipzig (313)

Volkmann, Hans-Erich, Prof. Dr., Direktor und Professor, MGFA $(243,304)$

Wagner, Armin, M.A., Oberleutnant, MGFA (207)

Walzl, August, Dr., Schmalweg 5, A-9241 Wernberg (277)

Wette, Wolfram, Wiss. Oberrat, Dr., MGFA (225)

Wippich, Rolf-Harald, Prof. Dr., Sophia University, 4 Yonban-cho, Chiyoda-ku, Tokyo 102, Japan (259) 